\title{
Distinguishing Different Levels of Consciousness using a Novel Network Causal Activity Measure
}

\author{
Nikita Agarwal \\ Consciousness Studies Programme \\ National Institute of Advanced Studies \\ Bengaluru, India \\ nktagarwal01@gmail.com
}

\author{
Aditi Kathpalia \\ Consciousness Studies Programme \\ National Institute of Advanced Studies \\ Bengaluru, India \\ kathpaliaaditi@nias.res.in
}

\author{
Nithin Nagaraj \\ Consciousness Studies Programme \\ National Institute of Advanced Studies \\ Bengaluru, India \\ nithin@nias.res.in
}

\begin{abstract}
Characterizing consciousness, the inner subjective feeling that is present in every experience, is a hard problem in neuroscience, but has important clinical implications. A leading neuro-scientific approach to understanding consciousness is to measure the complex causal neural interactions in the brain. Elucidating the complex causal interplay between cortical neural interactions and the subsequent network computations is very challenging. In this study, we propose a novel quantitative measure of consciousness - Network Causal Activity - using a recently proposed Compression-Complexity Causality measure to analyze electrocorticographic signals from the lateral cortex of four monkeys during two states of consciousness (awake and anaesthesia). Our results suggest that Network Causal Activity is consistently higher in the awake state as compared with anaesthesia state for all the monkeys.
\end{abstract}

Index Terms-Network causal activity, causal density, Compression-Complexity Causality, consciousness, neuroscientific measures of consciousness

\section{INTRODUCTION}

Understanding Consciousness - the inner subjective feeling that is present in every experience (eg., in "seeing" a red rose, in the "feeling" of pain, in the "tasting" of tea etc.), is the final frontier of biomedical research. Defining, modeling and measuring consciousness is considered a hard problem [1]. Consciousness largely bounds two facets, namely, the "level" of consciousness and the "content" of consciousness. Experiences such as coma, different stages of anaesthesia and certain stages of sleep seem to indicate a loss of consciousness [2]. Quantitatively, consciousness can be featured as the distributed cortical activity in the sub-cortical regions of the brain relating to the conscious content at any instant. Qualitatively, consciousness is the most essential aspect of our daily experience as it plays a big role in decision making and adaptive planning [3].

Measuring consciousness is a great aid to clinical assessments as it helps in building computational and psychological models; and philosophical aspects to understand the principles connecting brain activity to consciousness experience of wakeful individuals and individuals with physiological, pharmacological and pathological loss of consciousness. Recently, a number of scientific measures of consciousness have been proposed, each having their own theoretical and mathematical framework. We shall briefly describe a few of them here.
Tononi's Integrated Information Theory of Consciousness (IIT) is a leading scientific theory [4], [5] that conceptualizes the criteria for assessing the consciousness level of any system, quantitatively, as well as, qualitatively. According to IIT, if a system intrinsically possesses both integrated and differentiated states of information, then it is bound to possess some level of consciousness (indicated by the symbol $\Phi$ in the theory). Non-zero values of $\Phi$ conforms the system is in a conscious state. Neurobiologically, the number of connections of neurons in brain networks as well as their complex dynamical interactions contributes to the quantification of $\Phi$, not necessarily only the number of neurons. Perturbational Complexity Index (PCI) [6] is a theory-driven index formulated to evaluate the level of consciousness in a clinical scenario. In order to calculate PCI, the cortex of brain is perturbed with trans-cranial magnetic stimulation to invoke distributed activity in the thalamocortical brain networks. These spatiotemporal responses are then compressed to measure their algorithmic complexity which is normalized and calibrated to yield an index of consciousness level known as PCI. A high value of PCI indicates a high and significant amount of complex interactions of neural activity in cortical areas. Another measure of consciousness, Neural Complexity [7], aims to quantify the interplay between statistically independent (functionally segregated) and statistically interdependent (functionally integrated) neuronal groups in the brain. For any dynamical system, it is an information theoretic measure that captures the mutual information present between the different active subsets of the whole system [8]. Yet another measure of consciousness, known as Causal Density [9], [10], defines consciousness as the fraction of significant causal interactions in brain networks using Granger Causality measure [11]. In [12], [13], there is a review of other scientific measures of consciousness which are similar to the ones described here. The aforementioned measures are categorized under Complexity Theories of Consciousness (please see [14]).

In [15], a simple model of spectral Granger bivariate causality is applied to visualize the information flow between different parts of cortex for different states - conscious and unconsciousness induced by different means, in monkeys. This enabled the investigation of large-scale information flow and causal interactions specific to different frequency-modes in the 
brain. A switch in the frequency-mode of neural communication was found to characterize the difference between different levels of consciousness in monkeys.

In this study, we propose a novel, time domain, Network Causal Activity approach to discriminate different levels of consciousness. A recently proposed causality measure, Compression-Complexity Causality [16], which has been rigorously tested on simulated data for realistic scenarios as well as real-world data is used to formulate the measure. Instead of frequency domain analysis used in [15], we use this Network Causal Activity measure to differentiate between conscious and unconscious states in monkeys. The organization of the paper is as follows. In section II, we give a description of our proposed methodology, the data sets used, and define Network Causal Activity measure based on Compression-Complexity Causality which is then applied on the data. This is followed by a detailed analysis and discussion in section III. We conclude in section IV with future research possibilities.

\section{Materials And Methods}

\section{A. Subjects and Data Acquisition}

For our work, we have used a subset of the dataset from the study conducted by Yanagawa et al. [15] which is made available in the public server neurotycho.org (http:// neurotycho.org/) [17]. In their study, electrocorticographic (ECoG) signals sampled at $1 \mathrm{kHz}$ were recorded by a Cerebus data acquisition system (Blackrock, UT, USA) from the lateral cortex of four monkeys (George, Chibi, Su, Kin2) using 128 channels electrodes during different stages of sleep, wakefulness and anaesthesia on different days. A complete description about the experiment can be found in [15]. We have focused on only the awake (eyes-opened) and ketamine-medetomidine induced anaesthetized conditions.

\section{B. Dataset Description}

From the recorded neural data collected from the experiments of the study in [15], 3 non-overlapping windows of $5 \mathrm{~s}$ each (corresponding to 5000 time points) were extracted from 126 channels to construct a sustained network of neural interactions for all the four monkeys in two states - awake (eyes open, conscious state) and anaesthetized (drugged using Ketamine and Medetomidine, loss of consciousness state) condition. We excluded data from two channels (nos. 73 and 123) since these were found to be unsuitable for computation of causality values. The data was used in the acquired form without any re-referencing or pre-processing.

\section{Network Causal Activity}

Network Causal Activity (NCA) is proposed as a quantitative measure of consciousness to capture average (significant) causal influence activity between all the elements or subsystems of a given system. We use a recently proposed measure - Compression-Complexity Causality (CCC) [16] to estimate causal influences. CCC Toolbox made available as a part of Supplemental Material for [16] was used.
CCC is a time-domain, model-free measure that has been demonstrated to outperform the well-known Granger Causality [11] and Transfer Entropy [18] for several systems (stochastic and chaotic) under wide variety of scenarios, such as, presence of noise, uniform and non-uniform sampling and linear filtering. We shall first briefly describe CCC and then propose NCA using CCC.

For two given time series $A$ and $B, \mathrm{CCC}$ is formulated as follows:

$C C\left(\Delta A \mid A_{\text {past }}\right)=E T C\left(A_{\text {past }}+\Delta A\right)-E T C\left(A_{\text {past }}\right)$, $C C\left(\Delta A \mid A_{\text {past }}, B_{\text {past }}\right)=\operatorname{ETC}\left(A_{\text {past }}+\Delta A, B_{\text {past }}+\Delta A\right)-$ $\operatorname{ETC}\left(A_{\text {past }}, B_{\text {past }}\right)$,

$C C C_{B_{\text {past } \rightarrow \Delta A}}=C C\left(\Delta A \mid A_{\text {past }}\right)-C C\left(\Delta A \mid A_{\text {past }}, B_{\text {past }}\right)$,

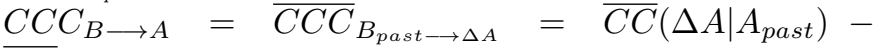
$\overline{C C}\left(\Delta A \mid A_{\text {past }}, B_{\text {past }}\right)$,

where $C C\left(\Delta A \mid A_{\text {past }}\right)$ is the compression complexity of time series $\Delta A$ (the current window with $w$ values from time series $A$ ) given time series $A_{\text {past }}$ (the window of $L$ values from the immediate past of $\Delta A$ ). This is estimated by first creating the time series $A_{\text {past }}+\Delta A$ (here ' + ' indicates the operation of appending at the end of the time-series) and then computing the difference between the Effort-ToCompress the appended time series $A_{\text {past }}+\Delta A$ and the Effort-To-Compress the time series of immediate past values $A_{\text {past }}$. $\operatorname{ETC}(x)$ is a complexity measure that captures the effort required by a lossless compression algorithm (known as Non-Sequential Recursive Pair Substitution Algorithm) to convert $x$ to a constant sequence [19]. ETC values are high for random sequences and low for periodic sequences. Similarly, $C C\left(\Delta A \mid A_{\text {past }}, B_{\text {past }}\right)$ refers to the compression complexity of time series $\Delta A$ given both the time series $A_{\text {past }}$ and $B_{\text {past }}$ (the immediate past values of $\Delta A$ taken from time series $A$ and $B$ ). This is computed by taking the difference between $\operatorname{ETC}\left(A_{\text {past }}+\Delta A, B_{\text {past }}+\Delta A\right)$ and $\operatorname{ETC}\left(A_{\text {past }}, B_{\text {past }}\right)$ where $\operatorname{ETC}(a, b)$ is the joint effort-tocompress complexity of time series $a$ and $b . C C C_{B_{\text {past } \rightarrow \Delta A} \text {, }}$, which is computed as a difference of two compression complexities $\left(C C\left(\Delta A \mid A_{\text {past }}\right)-C C\left(\Delta A \mid A_{\text {past }}, B_{\text {past }}\right)\right)$, is a measure of causality from the immediate past of $B$ to the current window of $A$. To compute the overall causality from time series $B$ to $A$ we take the average over all the temporal windows yielding $C C C_{B} \longrightarrow A$.

A statistically significant non-zero value of $C C C_{B} \longrightarrow A$ implies a causation from $B$ to $A$. A similar estimation of CCC from $A$ to $B$ can be computed $\left(C C C_{A \longrightarrow B}\right)$.

Having defined CCC for two time series, Network Causal Activity for multi-variate time series data $\mathbf{M}$ (with $m$ variables $^{1}$ ) is defined as the total average significant pairwise CCC values across all possible pairs. Mathematically,

$$
\operatorname{NCA}(\mathbf{M})=\frac{1}{n} \sum_{j, k=1, j \neq k}^{j, k=m} C C C_{j \rightarrow k}^{*},
$$

\footnotetext{
${ }^{1}$ If each of these series has $N$ time samples, then $\mathbf{M}$ is a $m \times N$ matrix. There would be $m^{2}-m$ pairwise CCC values out of which the highest $n=10 \%$ are taken as significant.
} 


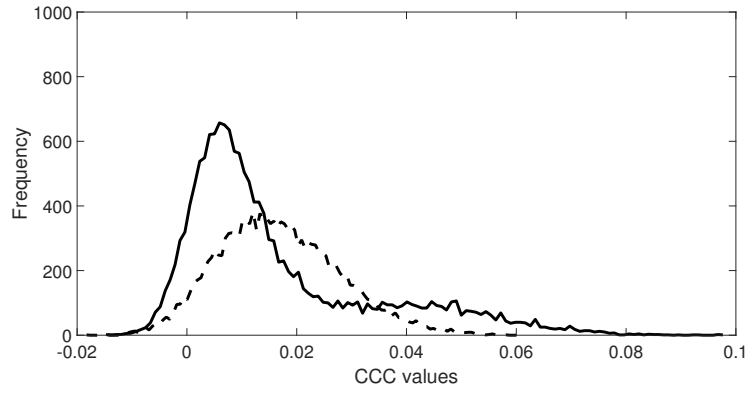

(a)

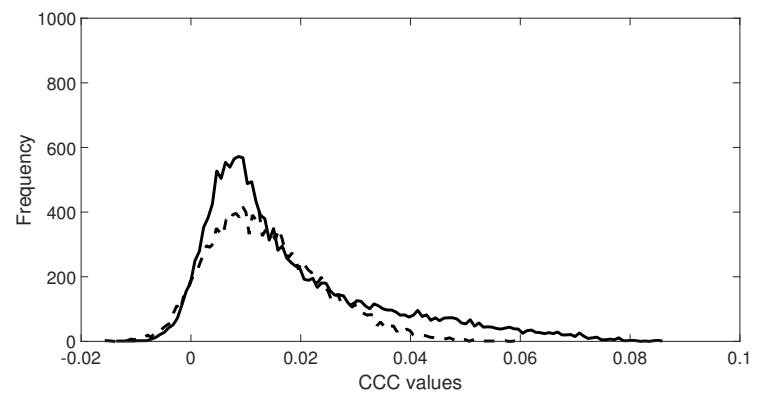

(c)

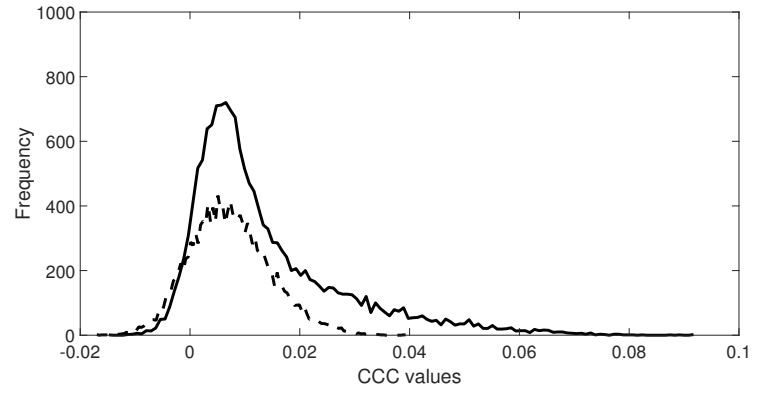

(b)

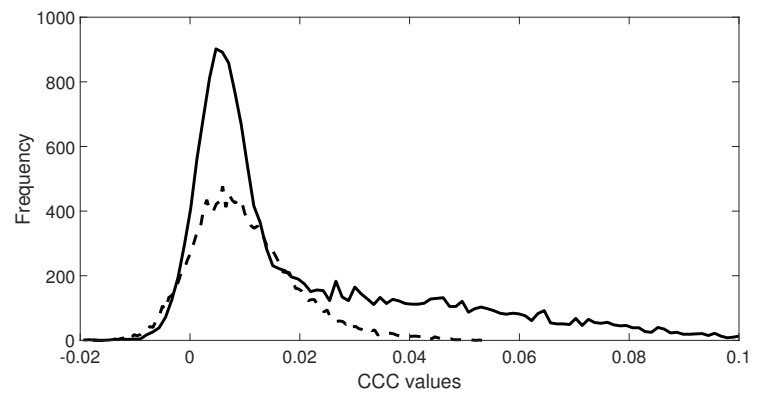

(d)

Figure 1: Histogram of pairwise CCC values across $126 \mathrm{ECoG}$ signal channels for each monkey for window $w_{1}$ (awake) and $w_{1}^{\prime}$ (anaesthesia): (a) George, (b) Chibi, (c) Su, (d) Kin2. Solid line (-) is for the Awake state and dotted line (- -) is for the Anaesthesia state. ECoG dataset obtained from [15].

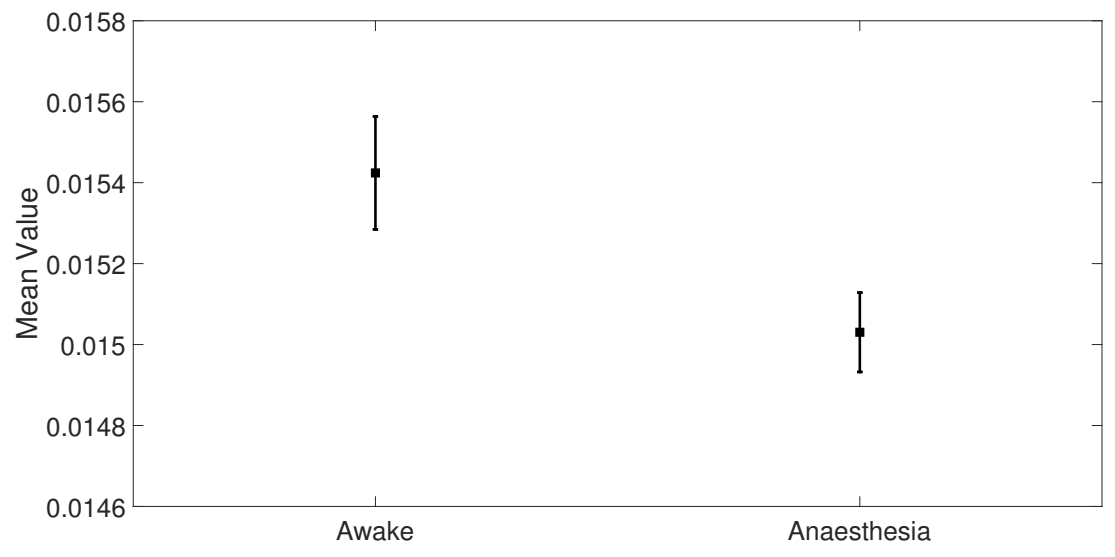

Figure 2: $95 \%$ confidence intervals for mean CCC values of pooled data of all windows (each of 5 second interval) of George accounting for 47, 250 samples, for awake as well as anaesthesia state showing a clear separation between the two.

where there are $n$ number of significant $\mathrm{CCC}$ values among all possible pairwise combinations of the $m$ variables. CCC value from the $j$-th time series to the $k$-th time series is said to be significant $\left(C C C_{j \rightarrow k}^{*}\right)$ if it is greater than some pre-defined threshold value $T$. Alternatively, we define significance as the highest $10 \%$ (in magnitude) of all pairwise CCC values for the given multi-variate time series. These two ways of defining significance will yield different values of NCA. We have used the latter in our study.

We estimated the pairwise CCC values for three non- overlapping windows of ECoG signals of 4 monkeys in Awake (conscious) and Anaesthesia (loss of consciousness) states. The settings that were chosen for estimating CCC are: $L=150, w=30, \delta=200$ (the step-size for the moving window), $B=2$ (number of bins ${ }^{2}$ ). These calculated CCC values are then used to estimate NCA using Eq.1 ( $N=5000$, $m=126, n=1575)$.

${ }^{2} \mathrm{CCC}$ is computed on a quantized version of the input real time series. The quantization is performed using uniform sized bins [16]. 
Table I: Mean and standard deviation of pairwise CCC values across 126 ECoG signal channels of different monkeys during awake state for 3 different windows, each of 5 seconds duration. ECoG dataset obtained from [15].

\begin{tabular}{|c|c|c|c|}
\hline \multirow{2}{*}{ Monkeys } & \multicolumn{3}{|c|}{ Awake } \\
\cline { 2 - 4 } & $w_{1}$ & $w_{2}$ & $w_{3}$ \\
\cline { 2 - 4 } & $0.0165 \pm 0.0176$ & $0.0139 \pm 0.0130$ & $0.0158 \pm 0.0153$ \\
\hline George & $0.0138 \pm 0.0139$ & $0.0159 \pm 0.0156$ & $0.0162 \pm 0.0142$ \\
\hline Chibi & $0.0183 \pm 0.0164$ & $0.0192 \pm 0.0168$ & $0.0107 \pm 0.0110$ \\
\hline Su & $0.0220 \pm 0.0241$ & $0.0246 \pm 0.0222$ & $0.0186 \pm 0.0196$ \\
\hline Kin2 & & & \\
\hline
\end{tabular}

Table II: Mean and standard deviation of pairwise CCC values across 126 ECoG signal channels of different monkeys during anaesthesia state for 3 different windows, each of 5 seconds duration. ECoG dataset obtained from [15].

\begin{tabular}{|c|c|c|c|}
\hline \multirow{2}{*}{ Monkeys } & \multicolumn{3}{|c|}{ Anaesthesia } \\
\cline { 2 - 4 } & $w_{1}^{\prime}$ & $w_{2}^{\prime}$ & $w_{3}^{\prime}$ \\
\cline { 2 - 4 } & $0.0168 \pm 0.0111$ & $0.0143 \pm 0.0107$ & $0.0140 \pm 0.0106$ \\
\hline George & $0.0073 \pm 0.0074$ & $0.0080 \pm 0.0084$ & $0.0088 \pm 0.0090$ \\
\hline Chibi & $0.0137 \pm 0.0104$ & $0.0106 \pm 0.0098$ & $0.0140 \pm 0.0116$ \\
\hline Su & $0.0099 \pm 0.0094$ & $0.0114 \pm 0.0099$ & $0.0096 \pm 0.0096$ \\
\hline Kin2 & & & \multicolumn{2}{|c|}{ CCean $(\mu) \pm$ Standard Deviation $(\sigma)$} \\
\hline
\end{tabular}

Table III: Network Causal Activity (NCA) estimates for all the monkeys for 3 different windows for both awake and anaesthesia states. The top $10 \%$ significant CCC values were used in computation of NCA. Mean NCA for awake state is higher than that of anaesthesia.

\begin{tabular}{|c|c|c|c|c|c|c|c|c|}
\hline \multirow{2}{*}{ Monkeys } & \multicolumn{4}{|c|}{ Awake } & \multicolumn{4}{c|}{ Anaesthesia } \\
\cline { 2 - 9 } & \multicolumn{3}{|c|}{ Network Causal Activity } & \multicolumn{3}{c|}{ Network Causal Activity } \\
\cline { 2 - 9 } & $w_{1}$ & $w_{2}$ & $w_{3}$ & Mean & $w_{1}^{\prime}$ & $w_{2}^{\prime}$ & $w_{3}^{\prime}$ & Mean \\
\hline George & 0.0564 & 0.0427 & 0.0482 & 0.0491 & 0.0378 & 0.0354 & 0.0355 & 0.0362 \\
\hline Chibi & 0.0460 & 0.0512 & 0.0477 & 0.0483 & 0.0214 & 0.0248 & 0.0263 & 0.0242 \\
\hline Su & 0.0555 & 0.0570 & 0.0356 & 0.0494 & 0.0344 & 0.0308 & 0.0391 & 0.0347 \\
\hline Kin2 & 0.0765 & 0.0727 & 0.0632 & 0.0708 & 0.0292 & 0.0307 & 0.0284 & 0.0294 \\
\hline
\end{tabular}

\section{ANALYSiS AND Discussion}

Mean and standard deviation of pairwise CCC values across 126 ECoG signal channels of 4 different monkeys for 3 different windows, each of 5 seconds duration, are given in Table I and Table II for the awake and anaesthesia states respectively. In Figure 1, histograms of pairwise CCC values for each monkey for window $w_{1}$ (awake) and $w_{1}^{\prime}$ (anaesthesia) are shown. In Table III, the Network Causal Activity (NCA) estimates are given for all the monkeys for 3 different windows for both the states. The top $10 \%$ significant CCC values were used in computation of NCA. From these tables, we can infer the following:

1) It is found that the standard deviations of CCC values in the awake state are consistently higher than that of 
the anaesthesia state in all the windows for all the monkeys (except for one window in case of monkey $\mathrm{Su}$ ). This finding implies that there are a higher number of differentiated causal neural interactions in the awake state as compared to anaesthesia state.

2) Mean NCA is consistently higher in awake state as compared to anaesthesia state across all the windows for all the monkeys. This is intuitive, since in the awake state we expect the average significant causal neural interactions to be of a higher magnitude.

3) The mean CCC values for awake state is significantly higher than the mean $\mathrm{CCC}$ value for the anaesthesia state. In order to substantiate this result statistically, a formal hypothesis '2 sample student's t-test' was performed for all the monkeys on data pooled over all the three windows of awake $\left(w_{1}, w_{2}\right.$, and $\left.w_{3}\right)$ as well as anaesthesia $\left(w_{1}^{\prime}, w_{2}^{\prime}\right.$, and $\left.w_{3}^{\prime}\right)$. The t-test results are summarized as follows:

- For George, the mean of awake state $(0.0154 \pm$ $0.0155)$ is significantly greater $\left(t_{94498}=-4.5272\right.$, $p=0)$ than that of anaesthesia state $(0.0150 \pm$ 0.0109).

- For Chibi, the mean of awake state $(0.0153 \pm$ $0.0146)$ is significantly greater $\left(t_{94498}=-93.7679\right.$, $p=0)$ than that of anaesthesia state $(0.0081 \pm$ 0.0083).

- For $\mathrm{Su}$, the mean of awake state $(0.0161 \pm 0.0155)$ is significantly greater $\left(t_{94498}=-38.0216, p=0\right)$ than that of anaesthesia state $(0.0128 \pm 0.0107)$.

- For Kin2, the mean of awake state $(0.0217$ $\pm 0.0222)$ is significantly greater $\left(t_{94498}=\right.$ $-103.0372, p=0)$ than that of anaesthesia state $(0.0103 \pm 0.0097)$.

A graphical analysis of this hypothesis test for 'George' is depicted in Figure 2.

\section{Conclusion}

Measuring Network Causal Activity, i.e., the average significant causal interactions in the brain, is a promising approach towards understanding consciousness. Our work demonstrates that Network Causal Activity, measured by estimating compression-complexity causality values of ECoG signals in monkeys can differentiate states of consciousness (awake vs. anaesthesia). Both, mean CCC and mean NCA measures are statistically significantly higher for awake state when compared with anaesthesia state. Going forward, it is worthwhile to estimate NCA for different stages of sleep and other states of consciousness (such as coma, vegetative state). Potentially, NCA could be further developed to provide robust measures of consciousness in clinical applications.

\section{ACKNOWLEDGMENT}

The authors gratefully acknowledge the financial support of 'Cognitive Science Research Initiative' (CSRIDST) Grant No. DST/CSRI/2017/54, 'Science and Technology for Yoga and Meditation' (SATYAM-DST) Grant No.
DST/SATYAM/2017/45(G) and Tata Trusts provided for this research. Aditi Kathpalia is thankful to Manipal Academy of Higher Education for permitting this research as part of the $\mathrm{PhD}$ programme.

\section{REFERENCES}

[1] David J Chalmers. Facing up to the problem of consciousness. Journal of consciousness studies, 2(3):200-219, 1995.

[2] Johan F Storm, Mélanie Boly, Adenauer G Casali, Marcello Massimini, Umberto Olcese, Cyriel MA Pennartz, and Melanie Wilke. Consciousness regained: disentangling mechanisms, brain systems, and behavioral responses. Journal of Neuroscience, 37(45):10882-10893, 2017.

[3] Anil K Seth, Eugene Izhikevich, George N Reeke, and Gerald M Edelman. Theories and measures of consciousness: an extended framework. Proceedings of the National Academy of Sciences, 103(28):1079910804, 2006.

[4] Giulio Tononi. An information integration theory of consciousness. BMC neuroscience, 5(1):42, 2004.

[5] Masafumi Oizumi, Larissa Albantakis, and Giulio Tononi. From the phenomenology to the mechanisms of consciousness: integrated information theory 3.0. PLoS computational biology, 10(5):e1003588, 2014.

[6] Adenauer G Casali, Olivia Gosseries, Mario Rosanova, Mélanie Boly, Simone Sarasso, Karina R Casali, Silvia Casarotto, Marie-Aurélie Bruno, Steven Laureys, Giulio Tononi, et al. A theoretically based index of consciousness independent of sensory processing and behavior. Science translational medicine, 5(198):198ra105-198ra105, 2013.

[7] Giulio Tononi, Olaf Sporns, and Gerald M Edelman. A measure for brain complexity: relating functional segregation and integration in the nervous system. Proceedings of the National Academy of Sciences, 91(11):50335037, 1994.

[8] Wayne Wu. The neuroscience of consciousness. In Edward N. Zalta, editor, The Stanford Encyclopedia of Philosophy. Metaphysics Research Lab, Stanford University, winter 2018 edition, 2018.

[9] Anil K Seth. Causal connectivity of evolved neural networks during behavior. Network: Computation in Neural Systems, 16(1):35-54, 2005.

[10] Anil K Seth, Adam B Barrett, and Lionel Barnett. Causal density and integrated information as measures of conscious level. Philosophical Transactions of the Royal Society A: Mathematical, Physical and Engineering Sciences, 369(1952):3748-3767, 2011.

[11] Clive WJ Granger. Investigating causal relations by econometric models and cross-spectral methods. Econometrica: Journal of the Econometric Society, pages 424-438, 1969.

[12] Nithin Nagaraj and Mohit Virmani. Is 'Information' fundamental for a scientific theory of consciousness? In Self, Culture and Consciousness, pages 357-378. Springer, 2017.

[13] Mohit Virmani and Nithin Nagaraj. A novel perturbation based compression complexity measure for networks. Heliyon, 5(2):e01181, 2019.

[14] Anil K Seth, Zoltán Dienes, Axel Cleeremans, Morten Overgaard, and Luiz Pessoa. Measuring consciousness: relating behavioural and neurophysiological approaches. Trends in cognitive sciences, 12(8):314321, 2008.

[15] Toru Yanagawa, Zenas C Chao, Naomi Hasegawa, and Naotaka Fujii. Large-scale information flow in conscious and unconscious states: an ecog study in monkeys. PloS one, 8(11):e80845, 2013.

[16] Aditi Kathpalia and Nithin Nagaraj. Data based intervention approach for complexity-causality measure. PeerJ Computer Science, 5(e196), 2019.

[17] Yasuo Nagasaka, Kentaro Shimoda, and Naotaka Fujii. Multidimensional recording ( $\mathrm{mdr}$ ) and data sharing: an ecological open research and educational platform for neuroscience. PloS one, 6(7):e22561, 2011.

[18] Thomas Schreiber. Measuring information transfer. Physical review letters, 85(2):461, 2000.

[19] Nithin Nagaraj, Karthi Balasubramanian, and Sutirth Dey. A new complexity measure for time series analysis and classification. The European Physical Journal Special Topics, 222(3-4):847-860, 2013. 